\title{
DYNAMIC CHANNEL SELECTION FOR MULTI-USER VIDEO STREAMING OVER COGNITIVE RADIO NETWORKS
}

\author{
Hsien-Po Shiang and Mihaela van der Schaar \\ Electrical Engineering, University of California Los Angeles, USA
}

\begin{abstract}
Due to the dynamic nature of cognitive radio networks, multi-user video streaming (with various video traffic characteristics and QoS requirements) requires efficient dynamic channel selection schemes to exploit available spectrum resources. To do this, a wireless user needs to effectively model the dynamic wireless environment and estimate the delay of video packet transmission when selecting a specific frequency channel. In this paper, we apply the priority virtual queue model for these wireless users to adapt their channel selection and maximize video qualities. The simulation results show that the proposed channel selection solution based on priority scheduling outperforms the conventional dynamic channel selection scheme by $2 \mathrm{~dB}$ (PSNR).
\end{abstract}

Index Terms - Video streaming, cognitive radio networks, priority queuing analysis.

\section{INTRODUCTION}

The emergence of cognitive radio networks have spurred both innovative research and ongoing standards [1][2]. Cognitive radio networks have the capability of achieving large spectrum efficiencies by enabling interactive wireless users to sense and learn the surrounding environment and correspondingly adapt their transmission strategies. Cognitive radio networks are generally considered highly dynamic due to the uncertainty of the primary users' behaviors that directly affects the spectrum opportunities available to the secondary users. Hence, secondary users need to model the other users and perform adaptive resource management in a distributed manner. However, for video streaming, a more sophisticated management mechanism is required to sustain the stringent delay and loss requirement. Importantly, a management scheme based on explicitly delay estimation of packet transmission using a certain frequency channel is necessary to satisfy the QoS requirements of the video streaming applications.

In this paper, we propose a dynamic channel selection scheme based on priority packet scheduling for multiple wireless users to transmit their delay-sensitive video packets over various frequency channels in the cognitive radio networks. Unlike prior works that seldom consider the requirement of the application layer [3], our solution explicitly considers various rate requirements and delay deadlines of different multimedia users. Specifically, we estimate the delay of sending video packets via various frequency channels using priority virtual queue analysis that considers 1) priorities of accessing the frequency channels, 2) different traffic loads/delay deadlines of different applications, and 3) different channel conditions in different frequency channels. Note that the priority queuing model allows the primary licensees to actively share the occupied channels instead of excluding all the other wireless users. However, by assigning highest preemptive priorities to the licensees (the primary users), the unlicensed users (the secondary users) do not impact the licensees.

The organization of the paper is as follows. In Section II, we present our problem formulation for multi-user video streaming over cognitive radio networks. In Section III, we show that the considered problem can be analyzed using priority virtual queue model and hence, facilitates the delay estimation for the proposed priority transmission scheduling solution. Section IV gives the simulation results and Section $\mathrm{V}$ concludes the paper.

\section{PROBLEM FORMULATION}

\subsection{Network settings and video traffic characteristics}

In this paper, we assume a single hop infrastructure in the cognitive radio networks. We assume that there are a total of $M$ frequency channels $\mathbf{F}=\left\{F_{1}, \ldots, F_{M}\right\}$ in the cognitive radio network and there are aggregated primary users $\mathbf{P U}=\left\{P U_{1}, \ldots, P U_{M}\right\}$ in each of the frequency channels. These primary users can only occupy their assigned frequency channels. Since the primary users are licensed users, they are provided with an interference-free environment [1]. We assume that there are $N$ secondary users $\mathbf{S U}=\left\{S U_{1}, \ldots, S U_{N}\right\}$ transmitting their own video applications in the system. These secondary users are able to schedule their video packets and transmit scheduled video packets using various frequency channels. Moreover, these secondary users are the license-exempt users, and hence, they cannot interfere with the primary users. 
We assume each $S U_{i}$ has a video application $V_{i}$. We separate each encoded video stream into a certain number of classes (quality layers) as in [6]. We assume that the packets within each video class have the same delay deadline. The number of priority classes for a video sequence $V_{i}$ equals $K_{i}$. We assume the total number of priority classes across all users in the network is $K$. The priority classes in the network are denoted as $C_{1}, \ldots, C_{K}$. For the purpose of analysis, we reserve the highest priority class $C_{1}$ for the primary users in each frequency channel, i.e. $\lambda_{1} \gg \lambda_{k}, 2 \leq k \leq K$. The video packets of all secondary users can be categorized into the rest of $K-1$ priority classes $\left(C_{2}, \ldots, C_{K}\right)$ to access the frequency channels. Hence, the total number of classes across all users in the network equals $K=\sum_{i=1}^{N} K_{i}+1$. We assume that the higher priority classes can preempt the transmission of the lower priority classes to ensure an interference-free environment for the primary users [7]. The priority reflects the ability of accessing the channel. Each video class $C_{k}$ is characterized by:

- $\quad \lambda_{k}$, the expected quality impact of receiving the packets in the class $C_{k}$. We prioritize the video classes based on this parameter. In the subsequent part of the paper, we label the $K$ classes (across all users) in descending order of their priorities, i.e. $\lambda_{1} \geq \lambda_{2} \geq \ldots \geq \lambda_{K}$.

- $\quad L_{k}$, the average packet lengths of the class $C_{k}$. The expected quality improvement for receiving a video packet in the class $C_{k}$ is defined as $\lambda_{k} \cdot L_{k}$.

- $\quad N_{k}$, the number of packets in the class $C_{k}$ in one GOP duration of the corresponding video sequence.

- $\quad P_{k}^{\text {succ }}$, the probabilities of successfully receiving the packets in the class $C_{k}$ at the destination. Thus, the expected number of the successfully received packets of the class $C_{k}$ is $N_{k} \cdot P_{k}^{\text {succ }}$.

- $D_{k}$, the delay deadlines of the packets in the class $C_{k}$. Due to the hierarchical temporal structure deployed in 3D wavelet video coders (see [6]), for a video sequence, the lower priority packets also have a less stringent delay requirement. This is the reason why we prioritize the video bitstream in terms of the quality impact. However, if the used video coder did not exhibit this property, we need to deploy alternative prioritization techniques $\lambda_{k}^{\text {video }}\left(\lambda_{k}, D_{k}\right)$ that jointly consider the quality impact and delay constraints.

At the client side, the expected quality improvement for video $V_{i}$ in one GOP can be expressed as:

$$
u_{i}=\sum_{C_{k} \in V_{i}} \lambda_{k} L_{k} N_{k} P_{k}^{\text {succ }},
$$

Here, we assume that the client implements a simple error concealment scheme, where the lower priority packets are discarded whenever the higher priority packets are lost [6]. This is because the quality improvement (gain) obtained from decoding the lower priority packets is very limited (in such embedded scalable video coders) whenever the higher priority packets are not received. Hence, we can write:

$$
P_{k}^{\text {succ }}=\left\{\begin{array}{l}
0 \quad, \text { if } P_{k^{\prime}}^{\text {succ }} \neq 1 \text { and } C_{k^{\prime}} \prec C_{k} \\
\left(1-P_{k}\right)=E\left[I\left(d_{k} \leq D_{k}\right)\right], \text { otherwise, }
\end{array}\right.
$$

where we use the notation $C_{k^{\prime}} \prec C_{k}$ to indicate that the class $C_{k}$ depends on $C_{k^{\prime}}$. Specifically, if $C_{k}$ and $C_{k^{\prime}}$ are classes of the same video stream, $C_{k^{\prime}} \prec C_{k}$ means $k^{\prime}<k$ due to the descending priority $\left(\lambda_{k^{\prime}}>\lambda_{k}\right) . P_{k}$ represents the end-to-end packet loss probability for the packets of class $C_{k} . d_{k}$ represents the experienced end-to-end delay for the packets of class $C_{k} . I(\cdot)$ is an indicator function. Note that the end-to-end probability $P_{k}^{\text {succ }}$ depends on the network resource, competing users' priorities as well as the deployed frequency channel selection.

\subsection{Channel selection strategy of the secondary users}

We denote the frequency channel selection of a secondary user $S U_{i}$ using $\mathbf{a}_{i}=\left[a_{i 1}, a_{i 2}, \ldots, a_{i M}\right] \in \mathcal{A}^{M}$, where $a_{i j} \in \mathcal{A}(\mathcal{A}=\{0,1\}) . a_{i j}=1$ represents the fact that the secondary user $S U_{i}$ chooses the frequency channel $F_{j}$. Otherwise, $a_{i j}=0$. Let $\mathbf{a}_{-i}$ denote the actions of the other secondary users except $S U_{i}$. Let $\mathbf{A}=\left[\mathbf{a}_{1}^{T}, \ldots, \mathbf{a}_{N}^{T}\right] \in \mathcal{A}^{M \times N}$ denote the total action set across all secondary users.

As stated in equation (1), each secondary user has its own video quality function as the utility function to maximize. Note that the utility function $u_{i}$ is also influenced by the action of other secondary users that select this frequency channel. In other words, the utility function should be regarded as $u_{i}\left(\mathbf{a}_{i}, \mathbf{a}_{-i}\right)$. Importantly, the secondary user $S U_{i}$ may not know the exact actions of other secondary users. It is unrealistic to assume that the exact action information can be collected timely to compute and maximize the actual utility function $u_{i}\left(\mathbf{a}_{i}, \mathbf{a}_{-i}\right)$. Hence, a more practical solution is to dynamically model the other secondary users' behavior by updating their probabilistic frequency selection strategy profile based on some available information exchange, and then maximizes the expected utility function of $S U_{i}$.

Hence, we define a frequency selection strategy profile of a secondary user $S U_{i}$ as a vector of probabilities $\mathbf{s}_{i}=\left[s_{i 1}, s_{i 2}, \ldots, s_{i M}\right] \in \mathcal{S}_{\alpha}=\mathcal{S}^{M} \quad, \quad$ where $\quad s_{i j} \in \mathcal{S}$ ( $\mathcal{S} \in[0,1]$ ) represents the probability of the secondary user $S U_{i}$ choosing the frequency channel $F_{j}\left(a_{i j}=1\right)$. Hence, the summation over all the frequency channels, $\sum_{j=1}^{M} s_{i j}=1$. Note that $s_{i j}$ can also be viewed as the 
fraction of data from $S U_{i}$ transmitted on frequency channel $F_{j}$, and hence, multiple frequency channels are selected for a secondary users with $s_{i j}>0$. Figure 1 illustrates the transmission strategies of the secondary users. Let $\mathbf{S}=\left[\mathbf{s}_{1}^{T}, \ldots, \mathbf{s}_{N}^{T}\right] \in \mathcal{S}^{M \times N}$ denote the total strategy profile across all the secondary users. The expected utility function, given a fixed strategy profile $\mathbf{S}=\left(\mathbf{s}_{i}, \mathbf{s}_{-i}\right)$ is

$$
U_{i}\left(\mathbf{s}_{i}, \mathbf{s}_{-i}\right)=E_{\left(\mathbf{s}_{i}, \mathbf{s}_{-i}\right)}\left[u_{i}(\mathbf{A})\right],
$$

where $\mathbf{s}_{-i}$ denotes the collected frequency selection profile. Then, the optimization problem becomes:

$$
\begin{aligned}
\mathbf{s}_{i}^{\text {opt }} & =\arg \max _{\mathbf{s}_{i}} U_{i}\left(\mathbf{s}_{i}, \mathbf{s}_{-i}\right) \\
& =\arg \max _{\mathbf{s}_{i}} \sum_{C_{k} \in V_{i}} \lambda_{k} L_{k} N_{k} P_{k}^{\text {succ }}\left(\mathbf{s}_{i}, \mathbf{s}_{-i}\right)
\end{aligned}
$$

\section{PRIORITY VIRTUAL QUEUING ANALYSIS FOR DELAY ESTIMATION}

In this section, we show how to calculate $P_{k}^{\text {succ }}\left(\mathbf{s}_{i}, \mathbf{s}_{-i}\right)$ in equation (4). Note that in our model in Figure 1, the packets from the primary users will not be seen at the physical queues of the secondary users, but only have impact on the virtual queues of the frequency channels. A wireless user needs to stream its video over an appropriate frequency channel to minimize the transmission delay $d_{k}$ and thereby, increase the video quality in equation (1). Different secondary users can experience different channel conditions using the same frequency channel. We denote $T_{i j}$ and $p_{i j}$ as the resulting physical transmission rate and packet error rate for the secondary user $S U_{i}$ transmitting through a certain frequency channel $F_{j}$.

We assume that the average rate requirement for the class $C_{k}$ traffic is $B_{k}$ (bit/s). Let $r_{j k}$ denote the average packet arrival rate of the class $C_{k}$ traffic using the frequency channel $F_{j}$. We have $r_{j k}=s_{i j} \times B_{k} / L_{k}, C_{k} \in V_{i}$. Since transmission errors are unavoidable in a wireless channel, we assume that packets will be retransmitted, if they are not correctly received. This can be regarded as a protection scheme similar to the Automatic Repeat Request protocol in IEEE 802.11 networks. Hence, the service time of the users can be modeled as a geometric distribution. Let $E\left[X_{j k}\right]$ and $E\left[X_{j k}^{2}\right]$ denote the first two moments of the service time of the class $C_{k}$ traffic using the frequency channel $F_{j}$. We have

$E\left[X_{j k}\right]=\frac{L_{k}}{T_{i j}\left(1-p_{i j}\right)}, E\left[X_{j k}^{2}\right]=\frac{L_{k}^{2}\left(1+p_{i j}\right)}{T_{i j}^{2}\left(1-p_{i j}\right)^{2}}, C_{k} \in V_{i}$

We modeled the virtual queues for a particular frequency channel using a priority $\mathrm{M} / \mathrm{G} / 1$ queue. Assume that the first two moment of the normalized loading of the

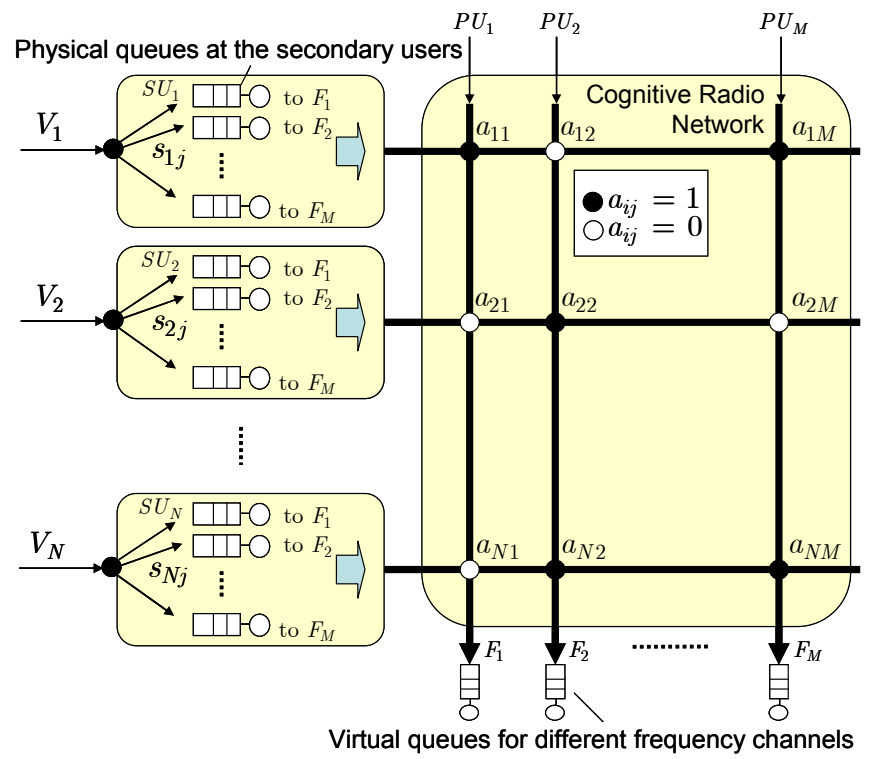

Fig. 1. Priroity transmission scheduling of the secondary users for each frequency channel.

primary user $P U_{j}$ is $\rho_{j}$ and $\rho_{j}^{2}$, respectively. By applying the Mean Value Analysis (MVA) in queuing theory [7], the average delay $E\left[\tilde{d}_{j k}\right]$ of the class $C_{k}$ traffic using the virtual queue of frequency channel $F_{j}$ becomes:

$$
\begin{array}{r}
E\left[\tilde{d}_{j k}\right] \cong \frac{\rho_{j}^{2}+\sum_{l=2}^{k} \mu_{j l}^{2}}{2\left(1-\rho_{j}-\sum_{l=2}^{k-1} \mu_{j l}\right)\left(1-\rho_{j}-\sum_{l=2}^{k} \mu_{j l}\right)}, \\
\text { where } \mu_{j k}=r_{j k} E\left[X_{j k}\right], \mu_{j k}^{2}=r_{j k} E\left[X_{j}^{2}\right] .
\end{array}
$$

By applying $\mathrm{M} / \mathrm{M} / 1$ queue at the physical queue, the probability of packet loss $P_{j k}\left(\mathbf{s}_{i}, \mathbf{s}_{-i}\right)$, for $C_{k} \in V_{i}$ becomes [7]:

$$
P_{j k}\left(\mathbf{s}_{i}, \mathbf{s}_{-i}\right)=r_{j k} \exp \left(-\frac{r_{j k}\left(1-r_{j k} E\left[\tilde{d}_{j k}\right]\right) D_{k}}{E\left[\tilde{d}_{j k}\right]}\right) .
$$

Note that $E\left[\tilde{d}_{j k}\right]$ depends on the strategies $\mathbf{s}_{-i}$ of other secondary users. Therefore, we can approximate the objective function in equation (4) for the video streaming of the secondary user $S U_{i}$ as (note that $\sum_{j=1}^{M} s_{i j}=1$ ):

$$
\underset{\mathbf{s}_{i}}{\operatorname{maximize}} \sum_{C_{k} \in V_{i}} \lambda_{k} L_{k} N_{k} \sum_{j=1}^{M} s_{i j}\left(1-P_{j k}\left(\mathbf{s}_{i}, \mathbf{s}_{-i}\right)\right) .
$$

\section{SIMULATION RESULTS}

We then simulate a larger number of secondary users and a larger number of frequency channels. First, we look at 
the case with 6 secondary users with video streaming applications ("Coastguard", frame rate of $30 \mathrm{~Hz}$, CIF format, $L_{k}=1000$ bytes and $D_{k}=500 \mathrm{~ms}$ ) sharing 10 frequency channels ( $N=6, M=10$ ). We compare the proposed Priority-Scheduling-based Channel Selection (PSCS) solving equation (7) with other two channel selection algorithms - the "Static Assignment" (SA) [5] and the "Dynamic Least Interference" (DLI) [4]. In the SA algorithm, a secondary user will statically select a frequency channel with the best effective transmission rate without interacting with other secondary users. In the DLI algorithm, a secondary user will dynamically select a single frequency channel that has the least interference from the other users disregarding the requirements of applications. We compute the average the video PSNR and the standard deviation of the PSNR over 100 simulation results in Table I for the video applications. The results show that our solution outperforms the other two approaches for delay-sensitive multimedia applications in terms of packet loss rate (PLR) and video quality (PSNR) especially when the primary users are present. The standard deviations of our solution are also smaller than the other two approaches. Unlike the DLI that only considers how a single user adapts to the experienced environment, our solution allows the secondary users to consider the application requirements and optimize the receiving video qualities. The video streaming will be able to self-organize into various cognitive radio frequency channels while adapting to the video traffic characteristics and the channel conditions.

Next, we simulate the case with $N=20,22,25,27,30,40$ secondary users with video streaming applications. These secondary users share $M=10$ frequency channels. The empirical average $T_{i j}$ of the frequency channels is shown to be $3 \mathrm{Mbps}$, instead of $1 \mathrm{Mbps}$ in the previous simulation. Figure 2 show the average PSNR over the $N$ video streams (instead of over 100 times simulations in the previous case). Larger $N$ reduces the available resources that can be shared by the video streams, and hence, increases the application layer packet loss rate (due to the expiration of the delay deadline) and hence, decreases the received video quality. The results show that our solution outperforms the other two approaches for multi-user video streaming applications.

\section{CONCLUSIONS}

In this paper, we propose a dynamic channel selection scheme based on priority scheduling. We select frequency channels for prioritized video packets based on priority virtual queue analysis in cognitive radio networks. Importantly, unlike conventional channel allocation schemes that select the least interfered channel merely based on the channel estimation, the proposed solution allows the secondary users to consider the application layer parameters, such as delay deadline and required rate, to maximize transmitted video qualities. The results show that our proposed solution outperforms the conventional dynamic channel allocation scheme.

\begin{tabular}{c|c|c|c|c}
\hline \hline & No Primary users & \multicolumn{3}{|c}{$\begin{array}{r}\text { Primary users randomly appear } \\
\left(\rho_{i}=0.25\right)\end{array}$} \\
\hline $\begin{array}{c}\text { Average } \\
T_{i j}=1 \mathrm{Mbps}\end{array}$ & $\begin{array}{c}\text { Average Y-PSNR } \\
(\mathrm{dB})\end{array}$ & $\begin{array}{c}\text { Average Y- } \\
\text { PSNR (dB) }\end{array}$ & PLR & $\begin{array}{c}\text { Y-PSNR } \\
\text { Standard } \\
\text { Deviation }\end{array}$ \\
\hline SA & 33.84 & 29.69 & $39.97 \%$ & 5.02 \\
\hline DLI & 33.90 & 30.37 & $33.85 \%$ & 4.41 \\
\hline PSCS & 35.61 & 32.36 & $18.76 \%$ & 2.26 \\
\hline \hline
\end{tabular}

Table I. Comparisons of the channel selection schemes for video streaming ( $N=6, M=10)$.

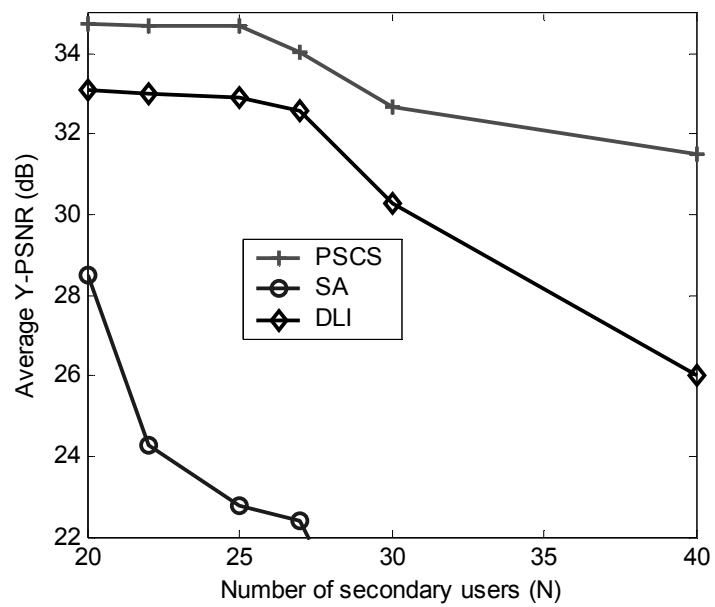

Fig. 2. Average Y-PSNR versus number of secondary users using different channel selection schemes.

\section{REFERENCES}

[1] S. Haykin, "Cognitive Radio: Brain-Empowered Wireless Communications," in IEEE Journal on Selected Areas in Communications, vol. 23, no. 2, Feb 2005.

[2] J. Mitola etal., "Cognitive radio: Making software radios more personal," IEEE Pers. Commun., vol. 6, no. 4, pp. 13-18, Aug. 1999.

[3] C. Cordeiro, K. Challapali, D. Birru and S. Shankar N, "IEEE 802.22: An Introduction to the First Wireless Standard based on Cognitive Radios," Journal of Communications, Academy Publishers, vol. 1, issue 1, Apr 2006.

[4] G. D. Kondylis and G. J. Pottie, "Dynamic Channel Allocation Strategies for Wireless Packet Access," IEEE VTC, Amsterdam, Sep 1999.

[5] S. Tekinay and B. Jabbari, "Handover and Channel Assignment in Mobile Cellular Networks," IEEE Communication Magazine, vol. 29, pp. 42-46, Nov 1991.

[6] M. van der Schaar, P. Chou, editors, Multimedia over IP and Wireless Networks, Academic Press, 2007.

[7] L. Kleinrock, Queuing Systems Volume I: Theory, NY: Wiley-Interscience Publication, 1975. 